\title{
Effects of hyperbaric oxygen therapy on 32 cases of full-thickness skin grafting for the repair of defects on the hand and foot
}

\author{
Yanhua Zhou, Dong Wang* \\ Department of Medical Oxygen Therapy, The Third Affiliated Hospital of Inner Mongolia Medical University, Baotou, Inner \\ Mongolia, China
}

Received: June 12,2018

Accepted: August 8, 2018

Online Published: September 10, 2018

DOI: $10.14725 /$ dcc.v5n3p28

URL: http://dx.doi.org/10.14725/dcc.v5n3p28

\begin{abstract}
Full-thickness skin grafting is one of the most commonly used repair methods for skin defects. It has the advantage of wearresistant with less scar hyperplasia and less difficult to operate with no symptoms. Hyperbaric oxygen was used in this study after full-thickness skin grafting from September 2012 to August 2016 to achieve the best effect of skin survival after surgery.
\end{abstract}

Key Words: Hyperbaric oxygen therapy, Skin defects, Full-thickness skin grafting

Hands and feet are important organs for weight-bearing and work. Skin and soft tissue defects often happen to those parts due to burns, accidents, surgery, etc., with a high incidence rate. $^{[1,2]}$ People have a high demand on the repair of this type of skin defects because of the important hand and foot function. Full-thickness skin grafting is one of the most commonly used repair methods. Compared with thin or intermediate thickness skin repair, it is wear-resistant with less scar hyperplasia. Meanwhile, in comparison to flap grafting, it is less difficult to operate with no symptoms (such as swelling) affecting the appearance. The blood circulation in the basal part of the wound may differ since the full-thickness skin is relatively thick, leading to the unsatisfactory skin survival and affecting the prognosis. In order to achieve the best effect of skin survival after surgery, on the basis of conventional treatment after full-thickness skin grafting, the author used hyperbaric oxygen in comprehensive treatment from September 2012 to August 2016. Now it was reported as follows.

\section{Data and methods}

\subsection{Clinical data}

From September 2012 to August 2016, 32 patients with skin defects on the hand and foot, who needed full-thickness free skin grafting, with no other complications, were admitted to the Third Affiliated Hospital of Inner Mongolia Medical University. They aged 5-63 (with an average age of 34.6), with the male-female ratio of 13:3. There were 13 cases of III degree thermal injury, 12 cases of scar contracture, 5 cases of car accident injury, 1 case of avulsion and 1 case of skin mass resection (confirmed as inflammatory granuloma by pathological examinations). As to the defect part, there were 11 cases of skin defects on the palm, 12 cases on the finger and fingerweb, 2 cases on the back of the hand, 4 cases on the sole of the foot and 5 cases on the ankle. The size of skin defect ranged from $(2 \mathrm{~cm} \times 3 \mathrm{~cm})$ to $(6 \mathrm{~cm} \times$ $11 \mathrm{~cm})$. They were divided into the conventional treatment

\footnotetext{
*Correspondence: Dong Wang; E-mail: nmbtwd@126.com; Address: Department of Medical Oxygen Therapy, The Third Affiliated Hospital of Inner Mongolia Medical University, Baotou, Inner Mongolia, China.
} 
group and the hyperbaric oxygen treatment group according to the condition that whether hyperbaric oxygen therapy was given after skin grafting, the general data of the two groups were comparable with no statistical significance $(p>.05)$.

\subsection{Methods}

These patients were divided into the conventional treatment group and the hyperbaric oxygen treatment group according to the odd and even numbers in the order of admission. The patients with scar hyperplasia, avulsion and skin masses underwent the surgery immediately after admission. Meanwhile, the patients with thermal injury underwent skin grafting in 7 days after injury, and the patients with granulation tissue formation caused by car accidents were given skin grafting in 3 weeks after injury. Hand anesthesia was performed with brachial plexus block, foot anesthesia was performed with continuous epidural block, and the anesthesia of the donor site was conducted with local infiltration anesthesia. The burn wounds were debrided to a healthy subcutaneous adipose tissue layer, the granulation tissues were stripped off from granulation tissues to the diploic layer, scar tissues were excised at the level of basal loose connective tissues, and basal necrotic tissues and foreign matters were removed during the treatment of avulsion, with nervevessel surgical exploration and basal hemostasis conducted. According to the size and shape of the recipient site, the fullthickness skin with the same size was taken from the skin on the abdomen, and the donor site was sutured by way of direct suture. The incised full-thickness free skin was grafted onto the wound surface, sutured, fixed, bound up and pressurized with the suture left. Anti-infective and symptomatic treatments were routinely given after surgery. For the hyperbaric oxygen treatment group, the patients were given conventional hyperbaric oxygen therapy for 7 days on the next day after surgery. Multi-place air-pressurized chamber (Yantai Moon Group: YC2680/0.3-16IV) was used to pressurize for $20 \mathrm{~min}$, with pressure increased to $0.20 \mathrm{MPa}$. A patient wore an oxygen face mask to breathe pure oxygen for $60 \mathrm{~min}$, with the intermittent time of $5 \mathrm{~min}$ during the process. And then the chamber finally depressurized the pressure to the atmospheric pressure at a constant rate for 20 min. Finally, the patient was taken out of the chamber. In the first 4 days, hyperbaric oxygen therapy was given twice a day; in the last 3 days, hyperbaric oxygen therapy was given once a day instead. On the $7^{\text {th }}$ day after surgery, the patients in the two groups were given a dress changing for the first time. The further treatment was conducted according to the healing of skin graft.

\subsection{Observed indicators}

(1) Calculate the survival rate of skin graft at the time of the first dressing change. The calculation formula is: the healing area of skin grafting divided by the area of skin grafting $\times 100 \%{ }^{[3]}$ The criteria of skin graft survival are that the blood vessels in the basal part of the wound grow into the skin graft, whose color turns red. (2) The average number of dressing change during hospitalization.

\subsection{Statistical treatment}

SPSS 17.0 was applied to data compilation and statistical analysis. The measurement data were represented by mean \pm standard deviation $(\bar{x} \pm \mathrm{s})$. The comparison in the survival rate of skin graft and the average number of dressing change between groups was made by use of $t$-test due to heterogeneity of variance. The difference $(p<.05)$ was of statistical significance.

\section{Results}

As to the survival rate of skin graft at the time of the first dressing change on the $7^{\text {th }}$ day after surgery, the hyperbaric oxygen treatment group was significantly higher than the conventional treatment group, and the difference was of statistical significance $(p<.05)$. With regard to the average number of dressing change during hospitalization, the hyperbaric oxygen treatment group was significantly lower than the conventional treatment group, and the difference was statistically significant $(p<.05$, see Table 1$)$.

Table 1: Comparison in the treatment effect between the conventional treatment group and the hyperbaric oxygen treatment group $(\bar{x} \pm \mathrm{s})$

\begin{tabular}{llll}
\hline Group & $\mathbf{n}$ & Survival rate of skin graft $(\%)$ & The average number of dressing change \\
\hline Conventional treatment group & 16 & $92.25 \pm 9.01$ & $4.69 \pm 1.92$ \\
Hyperbaric oxygen treatment group & 16 & $97.69 \pm 3.77^{*}$ & $3.50 \pm 0.82^{*}$ \\
$t$ value & & 2.23 & 2.27 \\
$p$ value & & .04 & .03 \\
\hline
\end{tabular}

Note. ${ }^{*} p<.05$ 


\section{Discussion}

After skin grafting, the skin cells survive in dependence on the interstitial fluids among the wound basal tissues in the recipient site. In 12 hours after skin grafting, the basal capillaries begin to grow into skin graft, and the new blood circulation between skin graft and the basal surface of the wound is rebuilt after 3-4 days. During this process, skin graft is in a lower oxygen tension, and the anoxic state affects the survival of skin graft. ${ }^{[4,5]}$ With the help of hyperbaric oxygen therapy, the oxygen content in the blood can be increased, so that the oxygen supply to skin graft in the basal part of the wound in the recipient site is enhanced consequently. ${ }^{[6]}$ In addition, hyperbaric oxygen therapy can accelerate the mitotic rate of epithelial cells, facilitate fibroblast synthesis and capillary proliferation and promote the survival of skin graft; ${ }^{[7]}$ it can kill some bacteria and increase the sensitivity of bacteria to antibiotics. ${ }^{[8]}$ This type of therapy can also enhance the function of macrophages and neutrophils ${ }^{[9]}$ to improve the anti-infective ability of the wound surface.

According to the treatment results, at the time of dressing change on the $7^{\text {th }}$ day after surgery, the survival rate of skin graft in the hyperbaric oxygen treatment group was significantly higher than that in the conventional treatment group. Upon leaving hospital, the average number of dressing change in the hyperbaric oxygen treatment group was

\section{References}

[1] Yao WT, Cai QQ, Wang JQ, et al. Comparison study on different flaps in repairing defect caused by resection of cutaneous malignant melanoma in the heel region. Chinese Journal of Reparative and Reconstructive Surgery. 2011; 25(7): 800-804. PMid: 21818943.

[2] Zhang WH, Zhou LP, Zhang ZH, et al. Treatment of infective refractory wound in burn patients by skin grafting combined with vacuum sealing drainage. Chinese Journal of Burns. 2014; 30(4): 365-367.

[3] Ye SJ, Zhang WZ, Pang SG, et al. Theraoeutic effect of Meek microskin-grafting technique in III burns over $80 \%$ total body surface area. Chinese Journal of Injury Repair and Wound Healing (Electronic Edition). 2013; 8(6): 35-38.

[4] Yang ZC. Burns Therapeutics. $3^{r d}$ ed. Beijing: People's Medical Publishing House; 2006. 463-464 p.

[5] Gibbs S, Silva Pinto AN, Murli S, et al. Epidermal growth factor and keratinocyte growth factor differentially regulate epi- significantly lower than that in the conventional treatment group. Through hyperbaric oxygen therapy, the survival rate of skin graft was improved with the number of dressing change reduced. This therapy also relieves patients of the economic burden and improves the therapeutic effect.

It should be noted that when performing the hyperbaric oxygen therapy: (1) Grasp the indications and contraindications of hyperbaric oxygen therapy; (2) Carry out this therapy on the next day after surgery with the stability of vital signs in peri-operative patients, no compulsory treatment allowed; (3) The application of hyperbaric oxygen therapy can not guarantee the survival of skin graft on a large area of hematoma, so complete hemostasis is the key to a successful surgery; (4) During the operation, the patient is not allowed to move around, and the stretcher should be provided to those patients with skin graft on the foot, in order to prevent the wound surface from re-bleeding; (5) Before a child patient is given hyperbaric oxygen therapy, the chamber is required to open only for him/her, with the maximum pressure increased to $0.16 \mathrm{MPa}$. After 3 to 4 times for adaptation, the adult pressure is used for the child's treatment.

\section{Conflicts of Interest Disclosure}

The authors have no conflicts of interest related to this article.

dermal migration, growth, and differentiation. Wound Repair Regent. 2000; 8(3): 192-203. https://doi.org/10.1046/j. 1524-475x.2000.00192.x

[6] Joan C, Phillips RN. Understanding hyperbaric oxygen therapy and its use in the treatment of compromised skin grafts and flaps. Plastic Surgical Nursing. 2005; 25: 72-80. https ://doi.org/10.1097/ 00006527-200504000-00006

[7] Yang R, Chen H, Wang YL. Promoting effect of hyperbaric oxygen on deep II degree burn wound healing in rats. Chinese Journal of Clinical and Experimental Pathology. 2009; 25(2): 187-191.

[8] Yi Z. Practical manual for technical specifications of hyperbaric oxygen therapy and management and quality control of hyperbaric oxygen chamber. Beijing: China Medical Science Press; 2007. 174$175 \mathrm{p}$.

[9] Wu JG, Jiang SF, Ding SW, et al. Effects of hyperbaric oxygen therapy on the survival of skin graft in a large-area burn. Chinese Journal of Nautical Medicine and Hyperbaric Medicine. 2013; 20(6): 423. 